\title{
A Qualitative Study of Chinese Hospital Nurse Leaders' Perceptions of Job Competency
}

\author{
Yang $\mathrm{Wu}^{1}$, Haiyun $\mathrm{Gai}^{2, *}$ \\ ${ }^{1}$ Shaanxi University of Traditional Chinese Medicine, Xianyang, Shaanxi 712046, China. \\ ${ }^{2}$ Xi'an Hospital of Traditional Chinese Medicine, Xi'an, Shaanxi 710021, China.
}

\begin{abstract}
How to cite this paper: Yang Wu, Haiyun Gai. (2021) A Qualitative Study of Chinese Hospital Nurse Leaders' Perceptions of Job Competency. International Journal of Clinical and Experimental Medicine Research, 5(2), 214-218. DOI: 10.26855/ijcemr.2021.04.016
\end{abstract}

Received: March 11, 2021

Accepted: April 5, 2021

Published: April 26, 2021

*Corresponding author: Haiyun Gai, Xi'an Hospital of Traditional Chinese Medicine, Xi'an, Shaanxi 710021, China.

Email: 2548513941@qq.com

\begin{abstract}
Objective: To understand the perceptions of nurse leaders in Chinese hospitals about job competency and to provide a basis for further constructing job competency for nurse leaders in Chinese medicine hospitals. Methods: A qualitative study was conducted to conduct semi-structured interviews and collect data from nurse leaders in Chinese hospitals, and the data were analyzed using the Colaizzi 7-step analysis method. Results: Five themes were extracted from the interviews, professional knowledge skills, communication and coordination skills, management and decision-making skills, learning ability and personal traits. Conclusion: The perceptions of Chinese hospital nurse leaders on job competency vary, and most of them believe that Chinese hospital nurse leaders, firstly, have solid theoretical knowledge of Chinese medicine and master the characteristic nursing techniques of Chinese medicine, while emphasizing management and communication skills, and finally, they should focus on personal learning ability and their own qualities.
\end{abstract}

\section{Keywords}

Chinese Medicine Hospitals, Chief Nurses, Job Competency, Qualitative Research

The rapid development of modern medicine has put forward higher requirements for the comprehensive ability of nurse leaders, and the job competency of nurse leaders refers to the comprehensive characteristics that distinguish excellent and general nurse leaders through the performance of ability, knowledge, skills and personal qualities in the work [1]. Domestic research on the competency of nurses and nurse leaders [2] is rich, but mostly focuses on general hospitals, and there is less research on the competency of nurse leaders in Chinese hospitals, but with the development of the Chinese medicine career requires more and more comprehensive quality of nurse leaders [3], so it is necessary to research on the job competency of nurse leaders in Chinese hospitals. In this study, we conducted in-depth interviews with clinical nurse leaders in Chinese hospitals to understand the perceptions and needs of nurse leaders in Chinese medicine hospitals about their job competencies, and to provide a theoretical basis for constructing a job competency model for nurse leaders in Chinese hospitals.

\section{Object and method}

\subsection{Study subjects}

A purposive sampling method was used to select 18 nurse leaders in a traditional Chinese medicine hospital in Shaanxi Province as the study subjects, including 8 new nurse leaders, age (33.20 \pm 1.30$)$, and working years

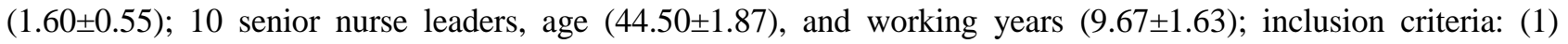
working in clinical positions of nurse leaders; (2) able to fully express their feelings, ideas and opinions; (3) volun- 
tary participation in this study and informed consent to the full recording.

\subsection{Research method}

\subsubsection{Data collection method}

A face-to-face, semi-structured in-depth interview method was used to collect data from July 2019 to August 2019. The researcher introduced himself before the interview and informed the interviewee of the purpose and significance of this interview, the interview location and time were discussed with the interviewee in advance (with the interviewee as the main focus) to ensure a quiet, comfortable, and undisturbed interviewed environment, and the entire interview process was recorded with two researchers, one asking questions and one taking notes, for 40 min-50 min, and the names were replaced with letters of the alphabet at the end of the interview. The interview outline was developed by reviewing competency-related literature: (1) What competencies do you think nurse leaders in TCM hospitals should have? The most important ones are? Talk specifically about: (2) What do you think nurse leaders do that would motivate/discourage you (assuming you nurse)? (3) How do you think you should communicate with the upper and lower departments? What help would you like from higher authorities? (4) Please tell us about the problems you have dealt with in your work? What did you do? Why did you do it? What were the results? (5) According to the changes in your career, you can talk about the performance, achievements and expectations at each stage of growth. (6) What has been the most profound feeling about the transition from nurse to nurse leader? (7) What education and years of experience do you think nurse leaders should have? (8) What do you think is the difference between a good nurse leader and an ordinary nurse leader? (9) Finally, what competencies should a nurse leader have in a Chinese medicine hospital?

\subsubsection{Analysis and organization}

After the interviews, two researchers repeatedly listened to the recorded data, converted the recordings into text, and organized and analyzed the interview data according to the Colaizzi phenomenological analysis [4-5].

\section{Results}

\subsection{TCM expertise skills Solid TCM knowledge and skilled TCM skills}

(1) Should have a wealth of clinical experience with proficient operational skills, each interviewee suggested that mastering nursing knowledge and skills as the head nurse is a basic requirement. Head nurse A: "professional skills must be strong, otherwise the nurse has a what cannot get, let you go over, cannot get is not a shame to the department”. Head nurse J. "When I can't tie the needle, the head nurse to help me technically, I especially recognized her". (2) Nurse leader should have professional sensitivity. D, F, G nurse leader: "If there is a new operation, it is definitely the head nurse to take the lead". $\mathrm{H}$ nurse leader: "Itself is a family of Chinese medicine or someone in the family engaged in Chinese medicine to understand better than those who have not received Chinese medicine study". B head nurse: "grasp the cutting-edge knowledge of Chinese medicine nursing at all times".

\subsection{Communication and coordination ability should have multidimensional communication ability}

(1) Nurses should listen more and encourage more. s head nurse: "I will observe each nurse's personality and work status, change the way of communication, for nurses who have never made a mistake and work seriously, occasionally have a mistake, will not be too serious to deal with, for personality big grin, make a mistake even small I will enlarge the treatment". C head nurse. "At that time, our department came to a new head nurse, I found some problems in the work, gave her some suggestions, she did not listen, I was quite sad". (2) The patient should be respected and think more differently. 1 head nurse: "The patient in small things are big things". O head nurse: "I usually deal with nurse-patient conflict, will stand in the patient's perspective, to give them the maximum benefit". (3) to superiors should be more cooperative, uploading. R head nurse: "to the notice issued by the nursing department, first understanding combined with the actual situation of the department before conveying down". K head nurse: "I often say in the department is that everyone has the time to need help from others, everyone has the opportunity to help others opportunity, so when things can help, help a little”.

\subsection{Management decision-making ability should have scientific and humane management ability}

(1) Rewards and punishments are clear. P nurse leader: "I reward those with high patient satisfaction to encourage nurses". (2) Treat things fairly and equitably and treat them equally. G nurse leader: "When I was a nurse before, there were colleagues who got along well, but after I became a nurse leader, I would be stricter with them instead". L nurse leader: "Contract and staff on staff are treated equally". (3) humanistic care. Q nurse leader: "to the 
nurses say words we are a pot of porridge, everyone is a part of it, the taste of porridge is decided by everyone”. E nurse leader: "I will encourage more contract nurses, contract nurses have no sense of belonging, lack of security, more encouragement can improve their self-confidence".

\subsection{Learning ability should have good learning ability}

(1) Learn scientific research thinking, scientific research ability. Chief nurse H: "to have a goal to learn, more learning, ideas and concepts to keep up". Chief nurse M: "in this environment (scientific research) in the hospital, the chief nurse must learn scientific research ability, with the department a piece of engagement." (2) "Go out, come in". I head nurse: "go out to learn, you can learn the good of other departments, but also see the shortcomings of their own departments". J head nurse: "head nurse to go out to learn more opportunities, we need to absorb more good".

\subsection{Personal traits should have non-powerful influence}

(1) Honest, kind and responsible. Nurse leader R: "give you the department, you have to be responsible, not something happened to say, it has nothing to do with me to escape". Nurse leader J: "kind, you can appreciate the pain of patients, only then have empathy". Nurse leader T: "Our department before a head nurse is a particularly good character, every day at work are cheerful, everyone is willing to say anything to her, including now retired, I am also willing to go and chat with her". (2) Self-discipline. D head nurse: "I asked the department nurses meeting, I usually arrive early, see me so early, some nurses are embarrassed to be late”.

\section{Discussion}

\subsection{Cognition of job competence among nurse leaders in Chinese hospitals}

It is easy to see from the perceptions of nurse leaders on job competency that most nurse leaders believe that TCM knowledge and practical skills are very helpful for management and also contribute to their own charisma. Bao Yue [6] conducted a study on 280 clinical nurses' practice skills competency, 30 of them conducted in-depth interviews and the other 250 conducted open-ended questionnaires, and the competency model derived from expert consultation through data analysis is as follows, with primary indicators (containing 7 items), secondary indicators (containing 31 items), and tertiary indicators (containing 93 items), in which the primary indicators of TCM knowledge and skills account for the greatest proportion of the first-level indicators is the knowledge and skills of Chinese medicine, which indirectly reflects the nursing staff's demand for career development, so it is presumed that the competency of the chief nursing officer in Chinese hospitals includes the knowledge and skills of Chinese medicine. Through this interview on the perceptions of Chinese hospital nurse leaders about their job competencies, it was initially concluded that Chinese hospital nurse leaders should have solid TCM knowledge and proficient TCM skills, multidimensional communication ability, scientific and humane management ability, learning ability and non-power influence.

\subsubsection{TCM expertise and skills are the expression of convincing power.}

Although the nurse leader is changing from a clinical position to a management position, it does not mean that the basic functions of the nurse leader should be discarded; on the contrary, the nurse leader should be strengthened on this basis. The results of a study by Hu Xiaoxia et al. [7] showed that the necessary skills of an excellent nurse leader are strong business skills, and the high level of expertise of the nurse leader increases the sense of security of nurses. The professional knowledge of nurse leaders can also improve their credibility and promote the development of the department.

\subsubsection{Good communication and coordination skills can close the distance between each other.}

Good communication ability is an important talent that nurse leaders have. Most nurse leaders believe that good communication and coordination ability makes it easy to manage the department without too much effort. Chen Yonghong et al. [8] concluded that effective communication can improve the patient experience, relieve patients' psychological pressure and improve the quality of care. When dealing with nurse-patient conflicts do two things, think differently and pay attention to grasp the proportion.

\subsubsection{Management decision making skills are demonstrated in the first level of quality control in the depart- ment.}

In this interview, six nurse leaders suggested that management competencies are expressed in the first level of quality control in the department [9], and the quality control check-ups in the department indirectly reflect the me- 
rits of nurse leaders' management. One study concluded that humane management, not only improves the job satisfaction of nurses, but also promotes teamwork and cohesion in the department [10]. From the interviews, it is clear that the management ability of the head nurse is actually managing people and the ward, which determines that communication with nurses and patients is very important.

\subsubsection{Good learning ability can master the cutting-edge knowledge and keep up with the development of TCM development.}

Sixteen nurse leaders in this interview mentioned learning ability, which should not be limited to professional knowledge, but also various aspects such as psychology and computer knowledge should be learned. The interviewees believe that through the learning of the head nurse can drive the development of the nursing team, stimulate the team effect and form the culture of the department.

\subsubsection{The personal traits of nurse leaders are the key factor in determining nursing team collaboration.}

During this interview, several nurse leaders suggested that the nurse leader's spirit of commitment and dedication. The spirit of commitment and dedication, which is a plus point for nurse leaders, not only improves the nurse-patient relationship, but also enhances nurses' support and confidence in the management of nurse leaders. Du et al. [11] concluded through in-depth interviews and expert correspondence that the personal traits of nurse leaders are closely related to job performance, so it is important to focus on the cultivation of personal traits of nurse leaders.

\subsection{Cultivation program for the job competency of nurse leaders in Chinese hospitals}

This interview with the head nurse provides the direction and target for the future training of the job competency of the head nurse in Chinese hospitals. From the results of this interview, it is known that the head nurse in Chinese hospitals should preferably have a background in Chinese medicine and have relatively strong learning and understanding of Chinese medicine knowledge, so in the training program of the head nurse in Chinese hospitals, the training of Chinese medicine knowledge and skills should be strengthened, and in addition to professional knowledge and skills, the head nurse should also be encouraged to strengthen her knowledge according to her ability, strengthen self-learning in other areas (psychology, computer, etc.), and hold regular TCM knowledge and skills competitions to exchange learning methods and experiences. In order to improve the research ability of nurse leaders, hospitals can stimulate their research talents through the selection of hospital-level projects. All six new nurse leaders mentioned mentorship during this interview [12], and a one-to-one mentorship training model for new nurse leaders can facilitate the role change of new nurse leaders, reduce the psychological pressure of new nurse leaders, and improve the management ability of new nurse leaders. Burnout was raised by four senior nurse leaders during this interview, which may be related to the stable development of the department, for which they should plan the career of nurse leaders [13], deploy their jobs appropriately, and enhance their career challenges.

\section{References}

[1] Ying Jusu, Liu Xiaoli, Wang Ling. (2019). A survey on the current situation of nurse leaders' job competency and training needs [J]. Chinese Journal of Modern Nursing, 2019(20): 2560-2564.

[2] Wen Xianxiu, Jing Jie, Lei Hua. (2015). Construction of the evaluation index system of nurse manager job competency [J]. Chinese Journal of Nursing, 2015, 50(02): 133-136.

[3] Huang Yao, Hu Jiale, Yu Leilei, Qian Peifen, Ruan Hong. (2016). The construction of domestic nurse leader competency model [J]. Journal of Liberation Army Nursing, 2016, 33(09): 28-31+35.

[4] Liu, M. Application of the seven steps of Colaizzi in the analysis of phenomenological research data [J]. Journal of Nursing, 2019, 34(11): 90-92.

[5] Yang, L., Yang, C.-Y., Ruan, H. (2019). Introduction and reflection on qualitative research report standards [J]. Journal of Nursing, 2019, 34(14): 105-108.

[6] Bao Yue. (2019). Competency-based modeling of the characteristic practice skills of nurses in Chinese hospitals [J]. Nursing Research, 2019, 33(13): 2221-2225.

[7] Hu Xiaoxia, Wang Sichen, Lu Liang, et al. (2014). Leadership skills expected of excellent ward nurse leaders [J]. China Nursing Management, 2014, 14(02): 177-179.

[8] Chen, Y. H., Wang, H., Zhu, Q. H., et al. (2020). A qualitative study of inpatient care experience [J]. Journal of Nursing, 2020, 35(07): 65-68.

[9] Miao, J. H., Lou, S., Sun, Xiaoyin, Fu, H. (2015). A practical study on behavior management of nurse leaders [J]. Chinese 
Journal of Nursing, 2015, 50(03): 273-275.

[10] Xu Yang, Zang Shuang. (2018). A qualitative study of imaging nurses’ perceptions of caring behaviors of nurse leaders [J]. Journal of China Medical University, 2018, 47(07): 646-649.

[11] Du Juan, Qi Yabin, Jiao Linlin. (2018). Construction of a training syllabus for nurse leader positions based on a competency model [J]. Chinese Nursing Education, 2018, 15(09): 645-649.

[12] Wang Huiying, Liu Yuping, Cui Yujie, et al. (2018). A qualitative study on the application of mentorship to the training model of new nurse leaders[J]. General Practice Nursing, 2018, 16(17): 2055-2058.

[13] Huang Xingzhi, Cheng Huiling, Pan Hui, Ning Xiaodong. (2015). Design and application of nurse leader training pathway [J]. Journal of Nursing Management, 2015, 15(02): 148-150. 